\title{
nature
}

nanotechnology

\section{Catalysis by design}

\section{A fundamental understanding of many factors — including composition, size, shape and surface structure - is vital for the development of new and improved catalysts.}

Catalytic reactions are a crucial component of our everyday lives, from their use in the chemical industry and the catalytic converters in our cars to the enzymatic processes that are central to the biochemistry of life. Given their importance, it is not surprising that the development of new catalysts - be they homogeneous or heterogeneous, biologically inspired or wholly synthetic - is a rich, diverse and everexpanding topic for research. Take, for example, organocatalysis: the use of small organic molecules to catalyse organic transformations has emerged over the past decade or so to become one of the most important methods for synthesizing chiral molecules ${ }^{1}$. Indeed, research into catalysis - and heterogeneous catalysis in particular - has the potential to be one of the most important and productive areas of nanoscience and technology in the next decade.

Heterogeneous catalysts lie at the heart of the chemical industry. They are typically composed of metal nanoparticles that are dispersed across the surface of a support material; in such catalysts the relationship between their structure and their properties (such as activity and selectivity) is finely poised. There are several benefits of reducing the size of the catalyst particles to the nanoscale, the most obvious being that the number of sites on the surface at which catalytic action can take place increases relative to the bulk. Moreover, the surface structure and electronic properties of the particle can change in ways that improve catalytic performance.

The benefits of moving to the nanoscale are perhaps best illustrated by gold. In its bulk form gold is relatively chemically inert, but in nanoparticle form it becomes active for a range of important catalytic reactions. These reactions include the oxidation of carbon monoxide to form carbon dioxide (as happens in catalytic converters), and the epoxidation of propene (an immensely important reaction, used in the production of numerous commercial materials, in which an oxygen atom is added to a molecule to convert a carbon-carbon double bond into a three-membered ring).

\section{There are several benefits of reducing the size of the catalyst particles to the nanoscale.}

The most recent work has revealed the possibility of working with even smaller gold nanoparticles. First, Turner and co-workers ${ }^{2}$ have shown that gold can carry out selective oxidation reactions using only molecular oxygen, whereas previously it was necessary for additives such as hydrogen or a peroxide species to be present as well. This behaviour only emerges in nanoparticles smaller than $2 \mathrm{~nm}$ in diameter, a regime that can be accessed with the help of techniques from cluster chemistry. Meanwhile Herzing and co-workers ${ }^{3}$ have used aberrationcorrected scanning transmission electron microscopy to examine gold clusters on iron oxide supports and found that the high activity of the catalyst could be related to the presence of gold clusters with diameters of just $0.5 \mathrm{~nm}$. Such clusters contain only 10 or so gold atoms.
To be able to analyse such catalysts in situ is an important, but challenging, endeavour. On page 598 of this issue, Paul Mulvaney and colleagues ${ }^{4}$ show that the catalytic performance of a single, isolated gold nanoparticle can be directly monitored using surface plasmon spectroscopy (see also p583). Elsewhere, Nolte and co-workers have shown that changes in the shape of rhodium nanoparticles supported on a magnesium oxide surface can be followed during oxidation and reduction reactions with in situ high-resolution X-ray diffraction ${ }^{5}$.

These papers highlight some of the very latest advances in nanoparticle catalysis; although in terms of understanding and design, work has only just begun. This is by no means easy - the performance of a catalyst can be affected by local differences in composition, size, shape and surface structure. But a fundamental understanding of all these factors is vital for the development of new and improved catalysts. What began with experiments on single-crystal surfaces in controlled environments has moved to ever-more intricate model systems and, now, to the study of real catalysts under reaction conditions. And through the molecular-level insights that these studies can provide, coupled with advances in the controlled synthesis of nanoparticles and nanoporous supports, precisely designed catalysts can emerge.

\footnotetext{
References

1. MacMillan, D. W. C. Nature 455, 304-308 (2008).

2. Turner, M. et al. Nature 454, 981-983 (2008).

3. Herzing, A. A., Kiely, C. J., Carley, A. F., Landon, P. \& Hutchings, G. J. Science 321, 1331-1335 (2008).

4. Novo, C., Funston, A. M. \& Mulvaney, P. Nature Nanotech 3, 598-602 (2008)

5. Nolte, P. et al. Science 321, 1654-1658 (2008).
} 